\title{
Structure for Synonymous Flow Label (SFL)
}

\author{
SUMIT KUSHWAHA \\ Electronics Engineering Department, \\ Kamla Nehru Institute of Technology, Sultanpur, INDIA.
}

\begin{abstract}
Because of the very high loss rate in general activity, enhancements in connection and transmission innovations have made it more difficult to evaluate packet loss utilizing dynamic execution estimation techniques with plotted traffic. That, along with seriously requesting administration level necessities, implies that network administrators currently should have the option to quantify the loss of the actual user data traffic utilizing inactive execution estimation strategies. Multiprotocol Label Switching (MPLS) strategy portrays the prerequisite for presenting stream characters inside the MPLS architecture. This paper depicts a strategy for achieving this by utilizing a method called Synonymous Flow Label (SFL) in which names that imitate the conduct of different labels give the recognizable proof assistance. These identifiers can be utilized to trigger per stream procedure on the packet at the receiving label switching router.
\end{abstract}

Keywords - Framework, SFL, MPLS, IPv6, Packet Loss, LSP, PHP, etc.

Received: August 14, 2020. Revised: October 6, 2020. Accepted: December 1, 2020. Published: December 31, 2020.

\section{Introduction}

Because of the very high loss rate in general activity, enhancements in connection and transmission innovations have made it more difficult to evaluate packet loss utilizing dynamic execution estimation techniques with plotted traffic. That, along with seriously requesting administration level necessities, implies that network administrators currently should have the option to quantify the loss of the actual user data traffic utilizing inactive execution estimation strategies. Multiprotocol Label Switching (MPLS) strategy sent should be straightforward to the end client, and it should be expected that they won't take any dynamic part in the estimation interaction. In reality, it is significant that any stream distinguishing proof method be undetectable to them and that no leftover of the estimation cycle spills into their organization. Furthermore, when there are numerous traffic sources, for example, in multipoint to point and multipoint to multipoint network conditions, there should be a technique whereby the sink can recognize packets from the different sources [1,2].

Modern networks if not oversubscribed, by and large drop generally couple of packets; consequently, packet misfortune estimation is exceptionally touchy to the regular division of the specific arrangement of packets to be estimated for misfortune. Without some type of shading or group checking, it may not be conceivable to accomplish the necessary precision in the misfortune estimation of client information traffic. In this way, when precise estimation of packet misfortune is required, it could be financially beneficial, or even be a specialized necessity, to remember some type of checking for the packets to appoint every packet to a specific counter for misfortune estimation purposes. At the point when this degree of exactness is required and the traffic between a source objective pair is liable to Equal Cost Multipath (ECMP), a boundary system is expected to assemble the packets into clumps. When a bunch is related at both entrance and departure, the packet bookkeeping system is then ready to work on the group of packets that can be represented at both the packet entrance and the packet departure [2]. Blunders in the bookkeeping are especially intense in Label Switched Paths (LSPs) exposed to ECMP on the grounds that the organization travel time will be distinctive for the different ECMP ways since:
- the packets may cross various arrangements of LSRs;

- the packets may leave from various interfaces on various line cards on LSRs; and

- the packets may show up at various interfaces on various line cards on LSRs.

A thought with this arrangement is Synonymous Flow Label (SFL) which show the cluster is the effect that this has on the way picked by the ECMP instrument. At the point when the individual from the ECMP way set is picked by profound packet assessment, a difference in cluster addressed by a difference in personality mark will no affect the ECMP way. In the event that the way part is picked by reference to an entropy name, at that point changing the cluster identifier won't bring about a change to the picked ECMP way. ECMP is so inescapable in multipointto-(multi)point networks that some strategy for trying not to account mistakes acquainted by ECMP needs with be upheld [3, $5]$.

\section{Synonymous Flow Labels}

An SFL is a name that causes the Egress Label Edge Router (LSR) to play out a formerly concurred activity notwithstanding handling and conveying the packet in the very same manner as the name that it is inseparable from (aside from if the activity says something else). The activity might be augmenting a counter, log a packet, or whatever else that is concurred between the MPLS peers. An SFL replaces, then again, actually it likewise causes at least one extra activity that have been recently concurred between the companion LER to be executed on the packet. There are numerous conceivable extra activities, for example, estimating the quantity of got packets in a stream, setting off an Internet Protocol Flow Information Export (IPFIX) catch, setting off different kinds of profound packet 
examination, or recognizing the packet source. For instance, in a Performance Monitoring (PM) application, the concurred activity could be recording the receipt of the packet by increasing a packet counter. This is a characteristic activity in numerous MPLS executions, and where upheld, this allows the usage of excellent packet misfortune estimation with no change to the packet sending framework $[4,7]$.

To represent the utilization of SFL, we start by considering the situation where there is an application name in the MPLS mark stack. Allow us to think about a pseudo wire (PW) on which it is wanted to make packet misfortune estimations. Two marks, inseparable from the PW names, are acquired from the departure Terminating Provider Edge (TPE). By switching back and forth between these SFLs and utilizing them instead of the PW name, the PW packets might be clumped for tallying with no effect on the PW sending conduct. The technique for acquiring these extra names is outside the extent of this content; notwithstanding, one control convention that gives a strategy for getting SFLs is depicted in $[5,6]$.

Then, consider a MPLS application that is multipoint to point, for example, a Virtual Private Network (VPN). Here, it is important to distinguish a packet bunch from a particular source. This is accomplished by making the SFLs source explicit, so that clumps from one source are stamped uniquely in contrast to clusters from another source. The sources all work freely and nonconcurrently from one another, autonomously organizing with the objective. Every entrance LER is consequently ready to set up its own SFL to recognize the sub stream and accordingly empower PM per stream [6].

At long last, we need to consider the situation where there is no MPLS application mark, for example, happens when sending IP over a Label Switched Path (LSP), i.e., there is a solitary name in the MPLS name stack. For this situation, presenting an SFL that was inseparable from the LSP mark would present organization wide sending state. This would not be worthy for scaling reasons. Along these lines, we must choose the option to present an extra mark. Where Penultimate Hop Popping (PHP) is being used, the semantics of this extra name can be like the LSP mark. Where PHP isn't being used, the semantics are like a MPLS Explicit NULL. In both of these cases, the mark has the extra semantics of the SFL [7].

\section{User Service Traffic in the Data Plane}

In this section, it is necessary to consider two cases $[8,11]$ :

a) Application Label Present

b) Single Label Stack

\subsection{Application Label Present}
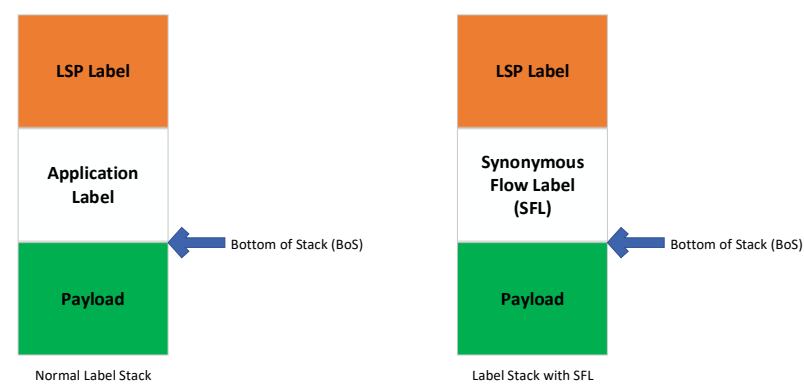

Fig. 1. Use of SFL in a Two Label MPLS Label Stack
Figure 1 shows the case wherein both a LSP name and an application mark are available in the MPLS name stack. Traffic with no SFL work present runs over the ordinary stack, and SFL empowered streams run over the SFL stack with the SFL used to demonstrate the packet cluster.

At the departure LER, the LSP mark is popped (if present). At that point, the SFL is prepared executing both the equivalent capacity and the comparing application work.

The TTL and the Traffic Class bits in the SFL Label Stack Entry (LSE) would regularly be set to a similar incentive as would have been set in the name that the SFL is inseparable from. In any case, it is perceived that, if there is an application need, these fields in the SFL LSE might be set to some other worth. A model would be the place where it was wanted to make the SFL trigger an activity in the TTL expiry special case way as a component of the mark activity.

\subsection{Single Label Stack}

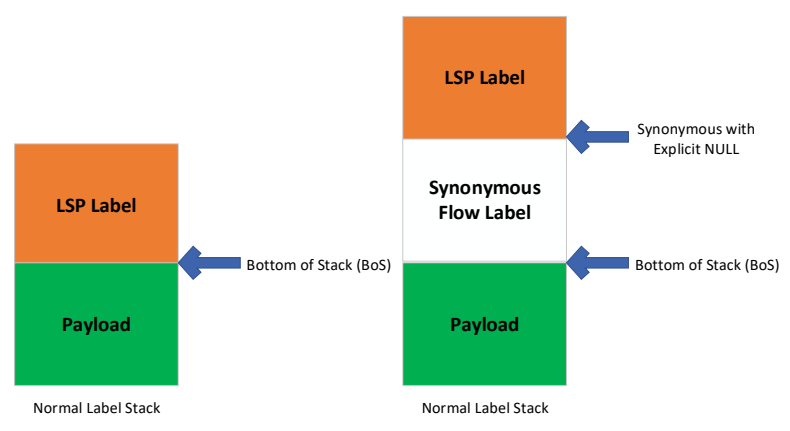

Fig. 2. Use of SFL in a Single Label MPLS Label Stack

Figure 2 shows the case where just a LSP mark is available in the MPLS name stack. Traffic with no SFL work present runs over the "ordinary" stack, and SFL empowered streams run over the SFL stack with the SFL used to show the packet bunch. Be that as it may, for this situation, it is vital for the entrance Label Edge Router (LER) to initially push the SFL and afterward to push the LSP mark.

At the accepting Label Switching Router (LSR), it is important to think about two cases:

a) where the LSP mark is as yet present

b) where the LSP name is penultimate bounce popped

On the off chance that the LSP name is available, it is handled precisely as it would typically be prepared, and afterward it is popped. This uncovers the SFL is just tallied and afterward disposed of. In this regard, the preparing of the SFL is inseparable from a MPLS Explicit NULL. As the SFL is the lower part of stack, the IP packet that follows is handled as would be expected.

In the event that the LSP mark is absent because of PHP activity in the upstream LSR, two practically identical handling moves can make place. The SFL can be dealt with either as a LSP mark that was not PHP and the extra related SFL move is made when the name is prepared or as a MPLS express NULL with related SFL activities. 


\section{Aggregation of SFL Actions}

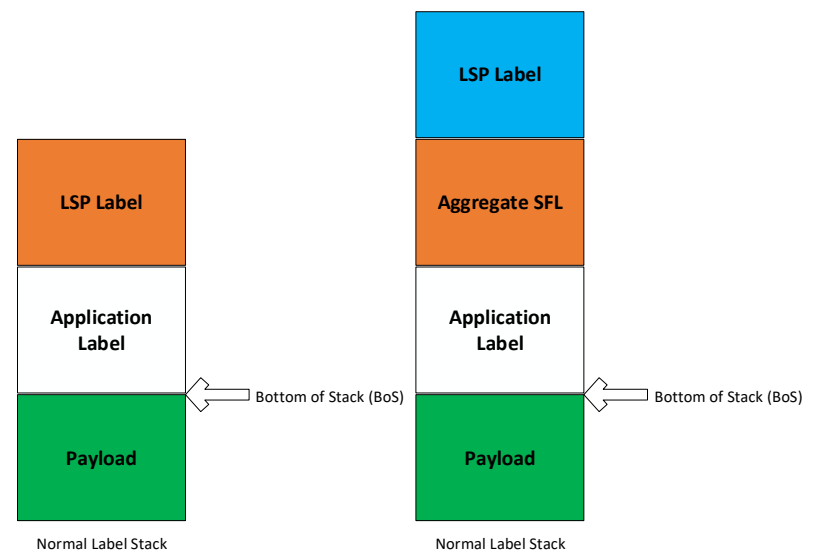

Fig. 3. Aggregate SFL Actions

It is attractive to total an SFL activity against various marks, for instance, where it is alluring to have one counter record the quantity of packets got over a gathering of use names or where the quantity of names utilized by a solitary application is enormous and the resultant expansion in the quantity of dispensed names expected to help the SFL activities may turn out to be too huge to possibly be practical. In these conditions, it is important to present an extra mark in the stack to go about as a total guidance. This isn't carefully an equivalent activity in that the SFL isn't supplanting a current mark yet is to some degree like the single name case, and a similar flagging, the executives, and setup instruments would be pertinent [9].

The total SFL is appeared in the mark stack portrayed in Figure 3 as going before the application name; be that as it may, the decision of position previously or after the application name will be application explicit. For this situation, the situating will rely upon whether the SFL activity needs the full setting of the application to play out its activity and whether the intricacy of the application will be expanded by finding an SFL following the application mark [10].

\section{Conclusion}

The acquaintance of an SFL with a current stream may make that stream take an alternate way through the organization under states of ECMP. This, thusly, may discredit certain employments of the SFL, for example, execution estimation applications. Where this is an issue, there are two arrangements worthies of thought: administrator may choose for consistently run with the SFL set up in the MPLS name stack, and administrator can choose for use entropy marks in an organization that completely underpins this kind of ECMP. On the off chance that this methodology is received, the mediating MPLS network should not load balance on any packet field other than the entropy mark. There are no new security issues related with the MPLS information plane. Any control convention used to demand SFLs should guarantee the authenticity of the solicitation, i.e., that the mentioning hub is approved to make that SFL demand by the organization administrator.

\section{References}

[1] Rosen, E., Tappan, D., Fedorkow, G., Rekhter, Y., Farinacci, D., Li, T., and A. Conta, "MPLS Label Stack Encoding", RFC 3032, DOI 10.17487/RFC3032, January 2001.

[2] Andersson, L. and R. Asati, "Multiprotocol Label Switching (MPLS) Label Stack Entry: "EXP" Field Renamed to "Traffic Class" Field", RFC 5462, DOI 10.17487/RFC5462, February 2009.

[3] Kompella, K., Drake, J., Amante, S., Henderickx, W., and L. Yong, "The Use of Entropy Labels in MPLS Forwarding", RFC 6790, DOI 10.17487/RFC6790, November 2012.

[4] Leiba, B., "Ambiguity of Uppercase vs Lowercase in RFC 2119 Key Words", BCP 14, RFC 8174, DOI 10.17487/RFC8174, May 2017.

[5] Bryant, S., Swallow, G., and S. Sivabalan, "A Simple Control Protocol for MPLS SFLs", Work in Progress, Internet-Draft, draft-bryant-mpls-sflcontrol-09, December 2020.

[6] Bryant, S., Ed. and P. Pate, Ed., "Pseudo Wire Emulation Edge-to-Edge (PWE3) Architecture", RFC 3985, DOI 10.17487/RFC3985, March 2005.

[7] Frost, D. and S. Bryant, "Packet Loss and Delay Measurement for MPLS Networks", RFC 6374, DOI 10.17487/RFC6374, September 2011.

[8] Claise, B., Ed., Trammell, B., Ed., and P. Aitken, "Specification of the IP Flow Information Export (IPFIX) Protocol for the Exchange of Flow Information", STD 77, RFC 7011, DOI 10.17487/RFC7011, September 2013.

[9] Farrell, S. and H. Tschofenig, "Pervasive Monitoring Is an Attack", BCP 188, RFC 7258, DOI 10.17487/RFC7258, May 2014.

[10] Fioccola, G., Ed., Capello, A., Cociglio, M., Castaldelli, L., Chen, M., Zheng, L., Mirsky, G., and T. Mizrahi, "Alternate-Marking Method for Passive and Hybrid Performance Monitoring", RFC 8321, DOI 10.17487/RFC8321, January 2018.

[11] Bryant, S., Pignataro, C., Chen, M., Li, Z., and G. Mirsky, "MPLS Flow Identification Considerations", RFC 8372, DOI 10.17487/RFC8372, May 2018 .

Creative Commons Attribution License 4.0 (Attribution 4.0 International, CC BY 4.0)

This article is published under the terms of the Creative Commons Attribution License 4.0

https://creativecommons.org/licenses/by/4.0/deed.en_US 

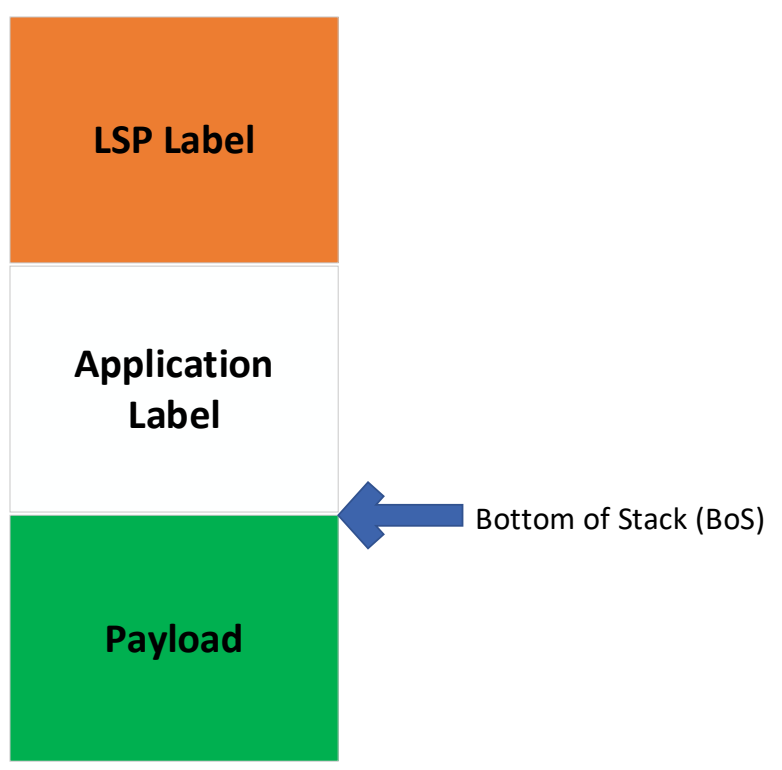

Normal Label Stack

Fig. 1. Use of SFL in a Two Label MPLS Label Stack

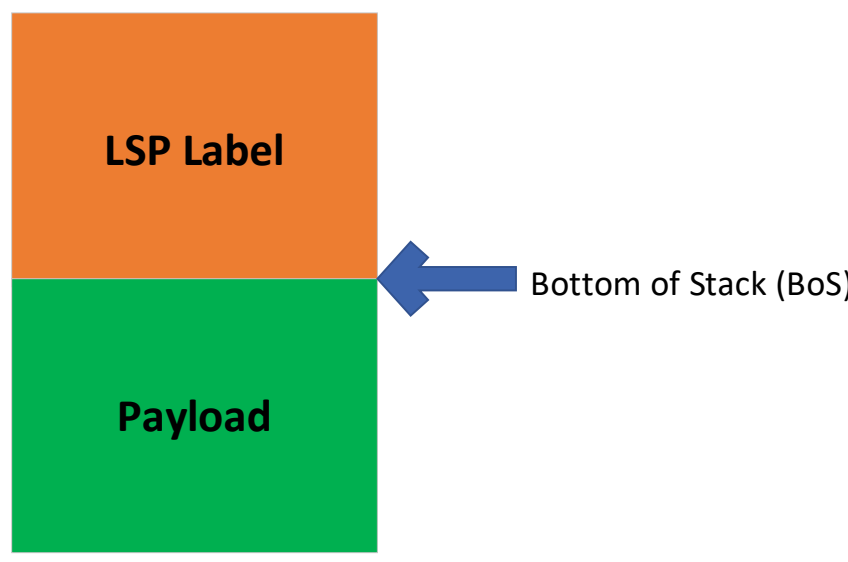

Normal Label Stack

Fig. 2. Use of SFL in a Single Label MPLS Label Stack

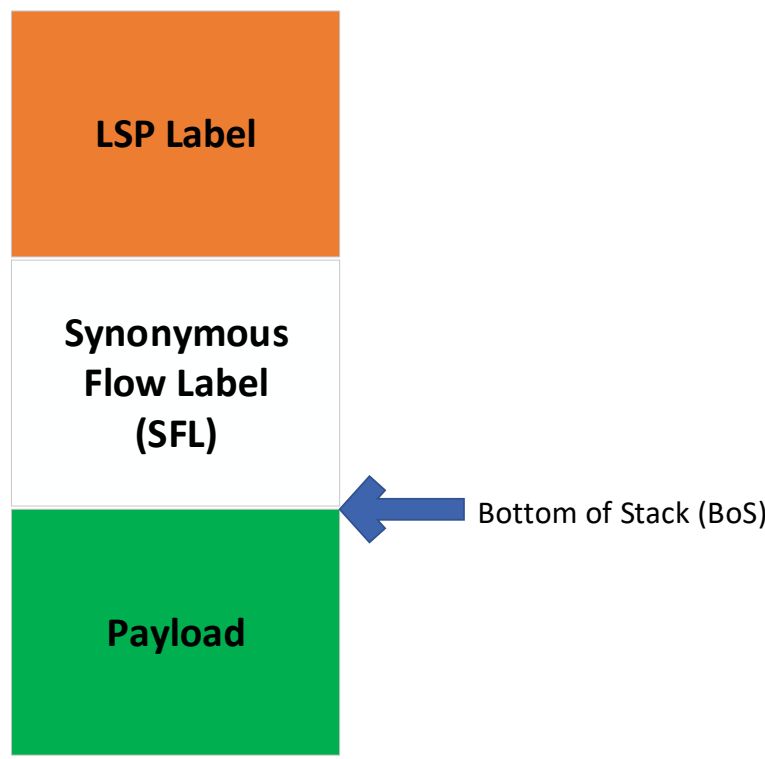

Label Stack with SFL

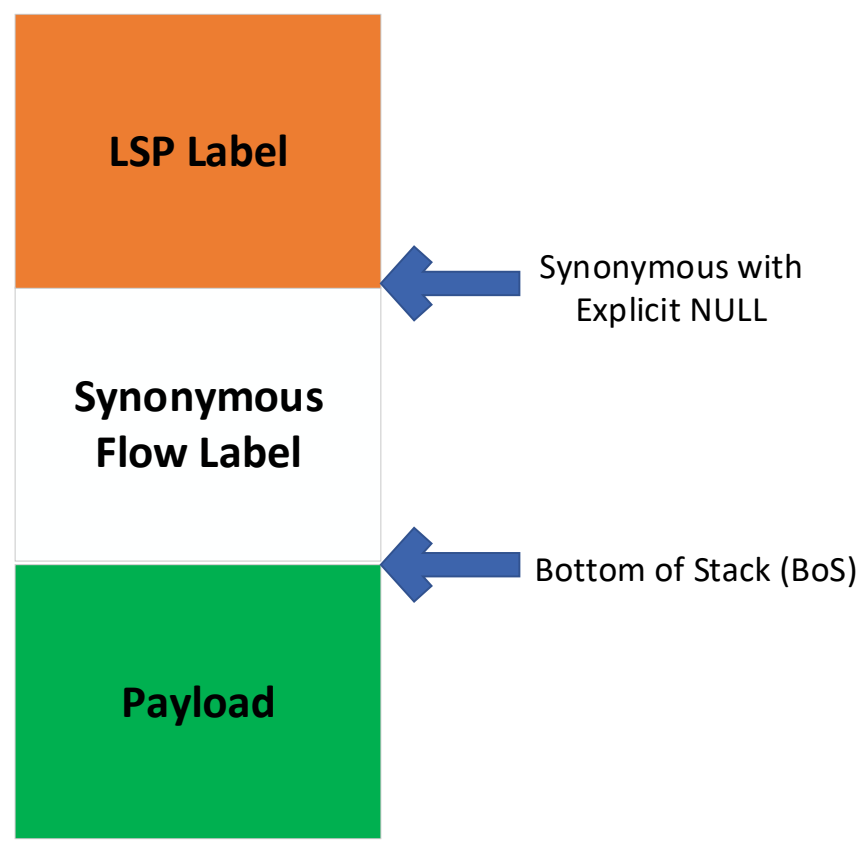

Normal Label Stack 


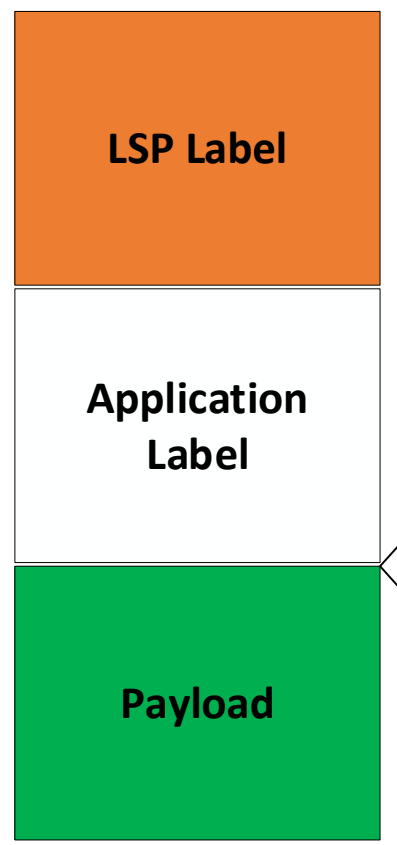

Normal Label Stack

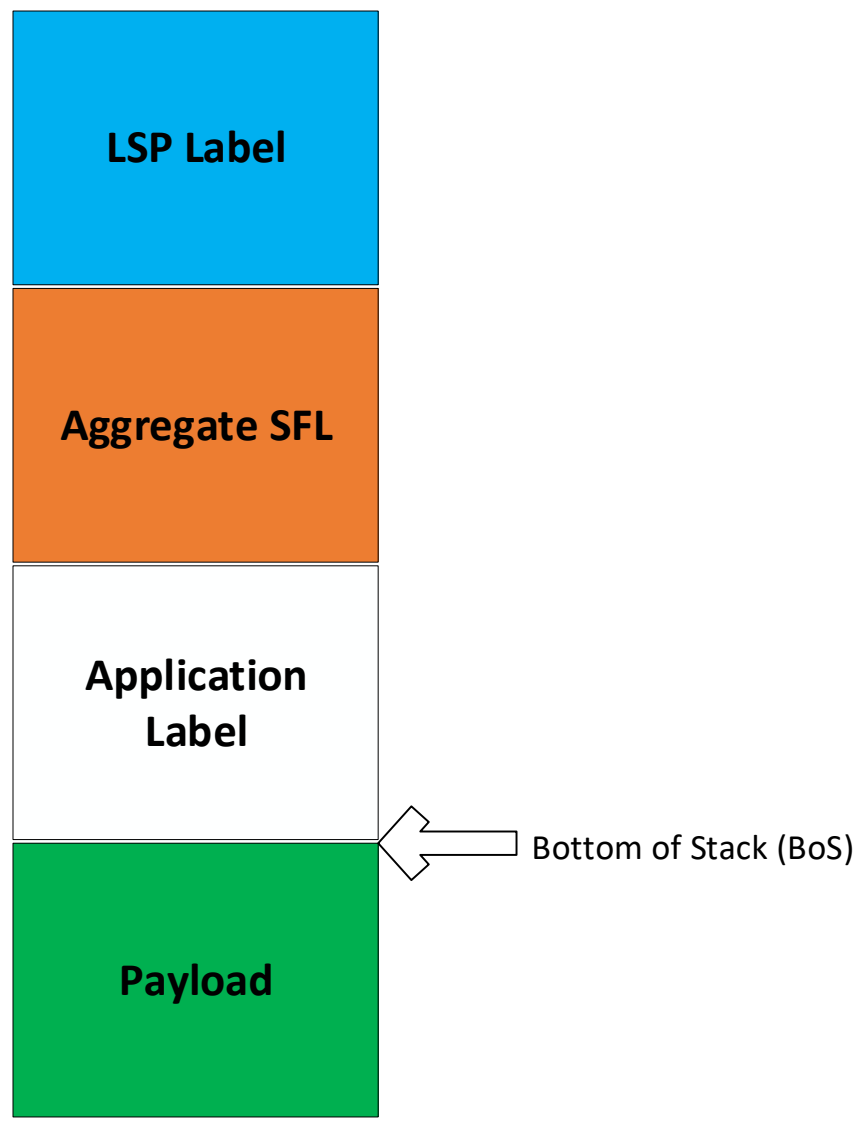

Normal Label Stack

Fig. 3. Aggregate SFL Actions 